\title{
The Science of Ray Tracing
}

\author{
Dhruv Dhote \\ B.Tech, CSE- GAG \\ Manav Rachna \\ International Institute of \\ Research and Studies \\ Delhi, India
}

\author{
Charu Virmani, PhD \\ Professor, CSE-FET \\ Manav Rachna \\ International Institute of \\ Research and Studies \\ Faridabad, India
}

\author{
K. Gopi Krishna \\ B.Tech, CSE -GAG \\ Manav Rachna \\ International Institute of \\ Research and Studies \\ New Delhi, India
}

\author{
Shivansh Raghav \\ B.Tech, CSE- GAG \\ Manav Rachna \\ International Institute of \\ Research and Studies \\ New Delhi, India
}

\begin{abstract}
The holy grail of rendering light and shadow in a scene by simulating and tracking every ray of light to blend in CG work with real-life scenes. It is the upcoming technology behind computer graphics for films and games to produce incredible realistic scenes in the computer generated world. The developers should utilize simulation for the precise prediction of the illuminance of the graphics to be used in films or games. This study digs the techniques and algorithms known for ray tracing and analyses the results Radiance and Lightscape 3.2 for a practical designed technique.
\end{abstract}

\section{Keywords}

Raytracing

\section{INTRODUCTION}

We ask that authors follow some simple guidelines. In essence, we ask you to make your paper look exactly like this document. The easiest way to do this is simply to download the template, and replace the content with your own material Ray tracing is the physics of line that tells us how the light ray bounces off the surface to cast shadows and reflections. It is an advanced way of producing realistic images that work by tracking and simulating light rays from a single source and generates realistic images to produce more lifelike shadows and reflections in movies and games. Traditionally, rasterization was used as it can be rendered quickly as compared to ray tracing but as technology is growing ray tracing has gained momentum and is used in movies and video games, Ray tracing primarily focuses on effects such as shadows and reflections which are difficult to simulate using other algorithms such as rasterization algorithms to reduce the render time. However, it might not yield good results in some cases. Images created with ray tracing are very detailed and realistic as it gives extra focus to each tiny detail which standard rasterization fails to achieve. Ray tracing distinguishes itself from other known algorithms in terms of graphics, shadows, textures, and draws the distance. Ray tracing has made animated images look close to real-life even old games like Minecraft look much better when ray tracing is applied. Moreover, the place where ray tracing stands out the most is the darker areas in a scene. Software such as blender, Autodesk Maya, unreal engine to name a few have achieved real-time ray tracing in scenes. Ray tracing is more visually appealing to users as it offers that realism in images that no other techniques can offer but it takes a huge time to render such images as it requires a powerful computer to achieve this. Nvidia has designed a ray-tracing graphics processing unit to handle these realistic images but is very expensive. To visually enhance an image and reduce the render time, ray tracing, and rasterization both are used in a scene to ensure the best quality. The light interacts as it would in real life emits a ray and this ray as a stream of photons travel in a single path resulting in absorption, refraction, and reflection would determine the intensity. Reflection would depict how the surfaces would look when light rays strike the surface and then reach the main camera in the scene. The advent of GPUs has adequate capability to explore new facets of ray tracing in the real-time and will employ rasterization and ray tracing as hybrid integration. This paper explores various tools and mechanisms of ray tracing and is divided into four sections. Section 1 introduces ray tracing, section 2 presents a detailed study about the tools and algorithm used in ray tracing, section 3 presents a comparison among known algorithms and tools and section 4 finally concludes.

\section{RELATED WORK}

In 2006 a film named CARS was the first animation film to use ray tracing developed by Pixar studios. It is the result of continuous efforts of Pixar of trials and errors to figure out how to make car moves according to the environment and to trace the reaction for each surface. It was a very complex process as it required a high computational complexity to render such images using ray tracing. The Pixar studios were able to achieve their goal and make the animation look very realistic and thus, the film turned into a massive success[911].

The first video game to implement real-time ray tracing was battlefield 5 Developed by DICE games and published by electronic arts released on 9 November 2018. Real-time ray tracing can be distinguished from normal ray tracing as video games require higher frame rates as compared to animated movies which require 24 frames per second. This was the time when real-time ray tracing was tested on video games as it was only used for making animated movies. The budget for the development was between 80 to 100 million dollars. It generated 1.29 billion dollars in the first quarter and a profit of 262 million dollars. It has sold up to 7.3 million copies till 2020.

But before the first video game, Nvidia developed their first ray-tracing graphics processor unit called RTX 2060 in 2018 to support real-time ray tracing in video games to make video games look more real soon. It was the era a graphics card was developed for real-time ray tracing and various versions of this graphics card Nvidia is continuously working to develop and enhance the capability for future projects. The following section highlights techniques of ray tracing to produce a big dive in AI rendering.

\subsection{Nvidia DLSS 2.0}

Artificial intelligence has revolutionized the gaming sector in gaming with advanced animation techniques and how AI reacts to video games. With Deep Learning Super Sampling 
(DLSS), NVIDIA is now set out to redefine real-time rendering through AI-based super-resolution - it renders few pixels and then uses Artificial intelligence to construct realistic and 4k images[12-16].

DLSS 2.0 is a new and improved technology in ray tracing that boosts frame rates while generating realistic images. It produces realistic without sacrificing the visual quality of the image and provides exponential better performance for users. Following are the advantages:-

- $\quad$ Superior image detailing - DLSS 2.0 offers a better image quality as compared to the native resolution which only renders one quarter to one-half of the pixels. It employs feedback techniques for sharp image detailing and improved stability from frame to frame.

- Great scaling across all GeForce RTX GPUs and resolutions - A new AI network that uses tensor Cores more efficiently to execute 2 times faster than the original. This improves frame rates per second and eliminates previous problems regarding performance and GPU usage issues.

- One network for the gamers - The original DLSS required an AI network for each latest game. DLSS 2.0 uses non-game specific content, therefore, delivering a generalized network that works across multiple games. This enhances faster game integrations and ultimately more games that support DLSS 2.0.

- $\quad$ Customizable options - DLSS 2.0 offers the best 3 image quality modes which are quality, balanced, and performance that controls the game's internal rendering resolution, with a better Performance mode that enables up to 4 times the super-resolution for eg: 4k. This means more user choice, and even much bigger performance boosts. Evolution of application program interface in ray tracing

GPUs evolved to become powerful rasterization machines. Adding programmability to architecture enabled complex rasterization based algorithms to be built $[17,18,19]$. This programmability enabled the GPUs to handle much more complex computational problems and eventually led to the launch of CUDA. NVIDIA's GPU-accelerated ray tracing efforts focused on ray-tracing through the CUDA programming model.

\subsection{Directx12 API in ray tracing}

In 2018, RTX graphics cards had launched with first graphics technologies: hardware-accelerated ray tracing. These technologies represented the biggest leap ever in the history of graphics since the invention of programmable shaders in 2002, bringing photorealistic reflections, shadows, and lighting to games. Microsoft has announced DirectX 12 Ultimate, their new graphics API, which will be used for nextgeneration gaming

DirectX 12 Ultimate will give developers a large, multiplatform to install base of hardware to target and ready-made tools. This makes game development much faster and easier and enables more developers to add more innovative technologies to their video games [20-23]. Many developers have crafted these new next-gen experiences featuring these technologies 30 DirectX games support ray tracing are being shipping or announced and now with the launch of DirectX 12 Ultimate, there will be highly used as their demand is increasing rapidly.

\subsection{Vulkan API in ray tracing}

Nvidia is trying to add Vulkan API for ray tracing for better performance as it gives higher frame rates in games and animations which can help in developing new things much easier as Vulkan API is suited for much better performance but developers are facing a huge challenge to achieve this as it is difficult to add Vulkan API in ray tracing and is not giving good results up till now so it could take a lot of time to develop but dlss 2.0 has boosted frame rates but if Vulkan API can be achieved in ray tracing it would be beneficial for the future development of ray tracing and it would achieve more quicker render time and more realistic images in less time.

\subsection{Geforce 20 series}

The NVIDIA GeForce RTX 20 series is a continuous series of graphics processing units that have been developed by Nvidia and it was revealed at Gamescom exhibition on August 20, 2018, and it had started shipping on 20 September 2018. It Served as the successor to the Nvidia GeForce gtx series the 20 series marked the introduction to Nvidia's Turing microarchitecture, and the first generation of RTX cards, the first in the industry to implement real-time ray tracing in video games and supporting various game engines. On July 2, 2019, the GeForce RTX Super line of cards was announced, which comprised of higher-spec versions of 2060 super 2070 super and 2080 super. Only These graphics are built for realtime ray tracing other companies have not launched graphics card for real-time ray tracing Nvidia will even announce more graphics card in the future as ray tracing is evolving in video games and in developing animation scenes in movies.

\subsection{RTX20 Series}

The RTX 20 series is based on the real-time ray tracing which is accelerated by the use of new RT cores, which are designed to speed up collision tests. The ray-tracing performed by RT cores can be used to produce effects such as reflections, refractions, shadows, depth of field, light scattering, and antialiasing. The second-generation Tensor Cores (succeeding Volta's) work in cooperation with the RT cores, and their AI features are used mainly to two ends: firstly, removing noise effects from a partially ray-traced image by detailing every ray cast; also another application of the Tensor cores is DLSS (deep learning super-sampling), a new method to replace antialiasing is by artificially generating high detail to upscale the rendered image into a higher resolution. Following are the new features in Turing:

$\begin{array}{ll}\text { - } & \text { DisplayPort 1.4a } \\ \text { - } & \text { PureVideo Feature video decoding } \\ \text { - } & \text { GPU Boost } \\ \text { VR support }\end{array}$

\section{MEDIUMS OF RAY TRACING}

Next-generation consoles are expected to support real-time ray tracing as it is the new emerging technology XBOX series $\mathrm{x}$ has promised to deliver a solid performance which can support $8 \mathrm{k}$ resolution and 60 frame rate per second and it would cost Rs.60000 and it has announced cloud gaming project $\mathrm{x}$ cloud so that it can also be experienced on smartphones and also PS5 has announced that it would also support ray tracing along with virtual reality support for nextgeneration consoles [24-26]. 
However, what remains to be seen is just how big of an impact real-time ray tracing will actually have and how widely it will be adopted by developers for PS5 and XBOX SERIES $\mathrm{X}$ games. Will the adoption of ray tracing be relegated to high-end PCs or Will it arrive on consoles is a huge question that everyone is asking both the consoles are set to release on summer 2020 but Nvidia's founder has said that next-gen laptop mobile GPU will have more power than nextgeneration consoles he demonstrated it at a launch event so AMD would have to be prepared to compete with Nvidia as they seem to have an upper hand.

Pixar created their renderer. Pixar's RenderMan renderer (PRMan). Which is a robust production renderer which they used for many CG movies and adding special effects PRMan uses the REYES scanline rendering algorithm which was added to PRMan by the developers to enable it to ray trace objects. When the new rendering algorithm was added to the PRMan, John Lasseter and his team started working on 'Cars', which turned out to be perfect testing ground and showcase for the ray-tracing functionality. There were two main rendering challenges in making the movie. Movie quality rendering of scenes of such complexity had only previously been possible with pure scaling rendering algorithms and was very time consuming as the render artists had to spend time for correcting lighting for each object in the scene separately $[27,28]$

But it was a massive success in movies and since then Pixar studios have made many movies using ray tracing they have improved lighting effects, shadows, and explosions in movies. Pixar is widely known to be one of the best-animated film making studios they have received many awards for their fantastic work ray-tracing brings that realism to objects and lighting that make them look spectacular [29].

Blender is used for making $3 \mathrm{~d}$ character models and objects but now it is enhanced with ray tracing it helps in making shiny surface and objects for a scene many developers use this tool to produce realistic animated movies, games and short movie clips moreover ray tracing is evolving in every software that involves the generation of $3 \mathrm{~d}$ character models because ray tracing is evolving rapidly in computer graphics as it gives developer to add much more details and they can gain much more revenue out of this but the only problem is that it requires a very powerful computer to implement ray tracing moreover the algorithm is much more complex than standard rasterization algorithm which makes it even more difficult to implement it practically but as technology is evolving so is ray tracing and blender is a very popular animation software it is widely used so it is being optimized for enabling ray tracing

\section{PROBLEMS ASSOCIATED WITH RAY TRACING}

The ray-tracing cards are not being completely utilized as many video games are not able to achieve real-time ray tracing so it is not all a very good choice to opt for ray tracing GPUs as many games are not able to achieve it and these graphics cards are way expensive to afford. Moreover, Nvidia RTX cards are being heavily criticized as it doesn't provide good frame rates per second in video games which make it a very bad deal although they can achieve realistic lighting and images but the major drawback is performance to counter that Nvidia is trying to use DLSS 2.0 which has given much better frame rates but it is still not up to the mark. Developers face a huge problem when they have to wait for render time and it makes the task much more complex.

In 2018 Nvidia demonstrated a short clip made using only real-time ray tracing but it took the developers to render a 2minute scene but the short clip was highly praised as it showcased what ray tracing could achieve the lighting was accurate it displayed realistic reflections following the concept of physics it would make many scenes look much better soon as new tools are being developed for implementing the same.

Ray tracing appears to be visually appealing but there are a lot of problems with this technology it requires high cost and computational power to generate such images. Sometimes it takes weeks to render even a single image because many operations have to be performed which makes it difficult to implement Moreover game developers are facing a huge challenge to implement real-time ray tracing in video games, .not many video games are supporting real-time ray tracing as it requires a lot of money and time to render images and they are sticking to simple rasterization technique as it is easy to implement and images can be quickly rendered also video games which can achieve real-time ray tracing are facing some issues as there are huge drops in frames which result in a terrible experience and it is discouraging developers to use real-time ray tracing in their video games moreover users are complaining about such horrible experience because of constant lag spikes and stuttering in their video games. So this means that performance is being impacted at the cost of producing realistic images which should not happen because without proper performance there would be no scope of this technology. Thus, the lack of support is making this technology difficult to be implemented and used by developers.

\section{SIMULATION ANALYSIS OF RAY TRACING}

Going to the test details, a $100 \mathrm{~m} 2$ atrium was modeled with a horizontal opening and conducted on the center of floor vertical wall. This is a very simple model called daylight factor estimation. It is done to illustrate the different methodology and to provide an estimation if the model is working. The well index of the model has come out to be in range of 0.5-2.5 using commercially available software. With overcast sky conditions, five ambient bounces (considering radiance) have been chosen. Simulation conducted gave rise to the below

1) Floor reflectance and atrium wall@0.2 and @0.4 respectively

2) Floor reflectance and atrium wall @0.2 and @0.8 respectively

Results are as shown in below figures 


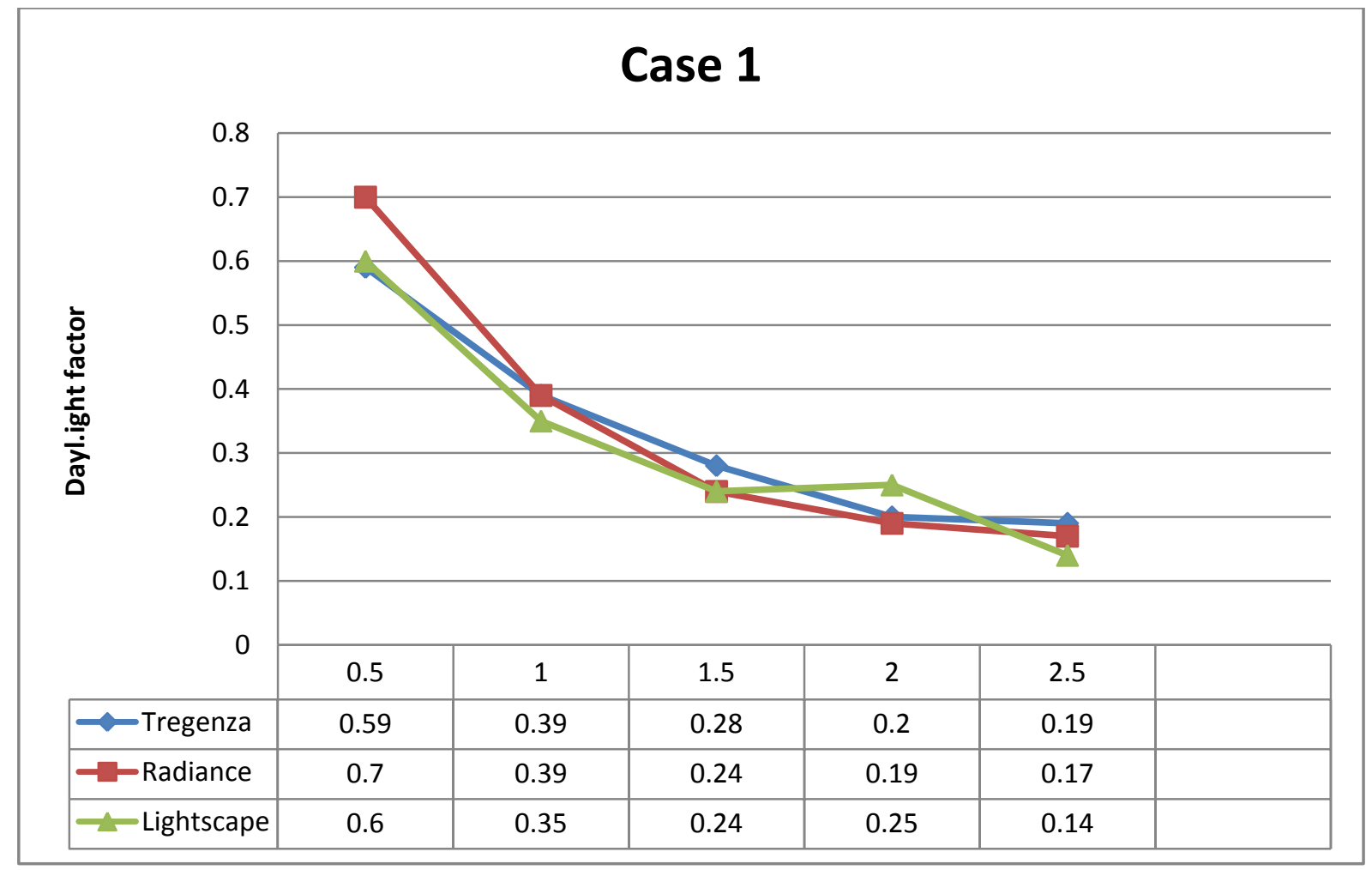

Figure 1: Comparison of horizontal DF for case 1

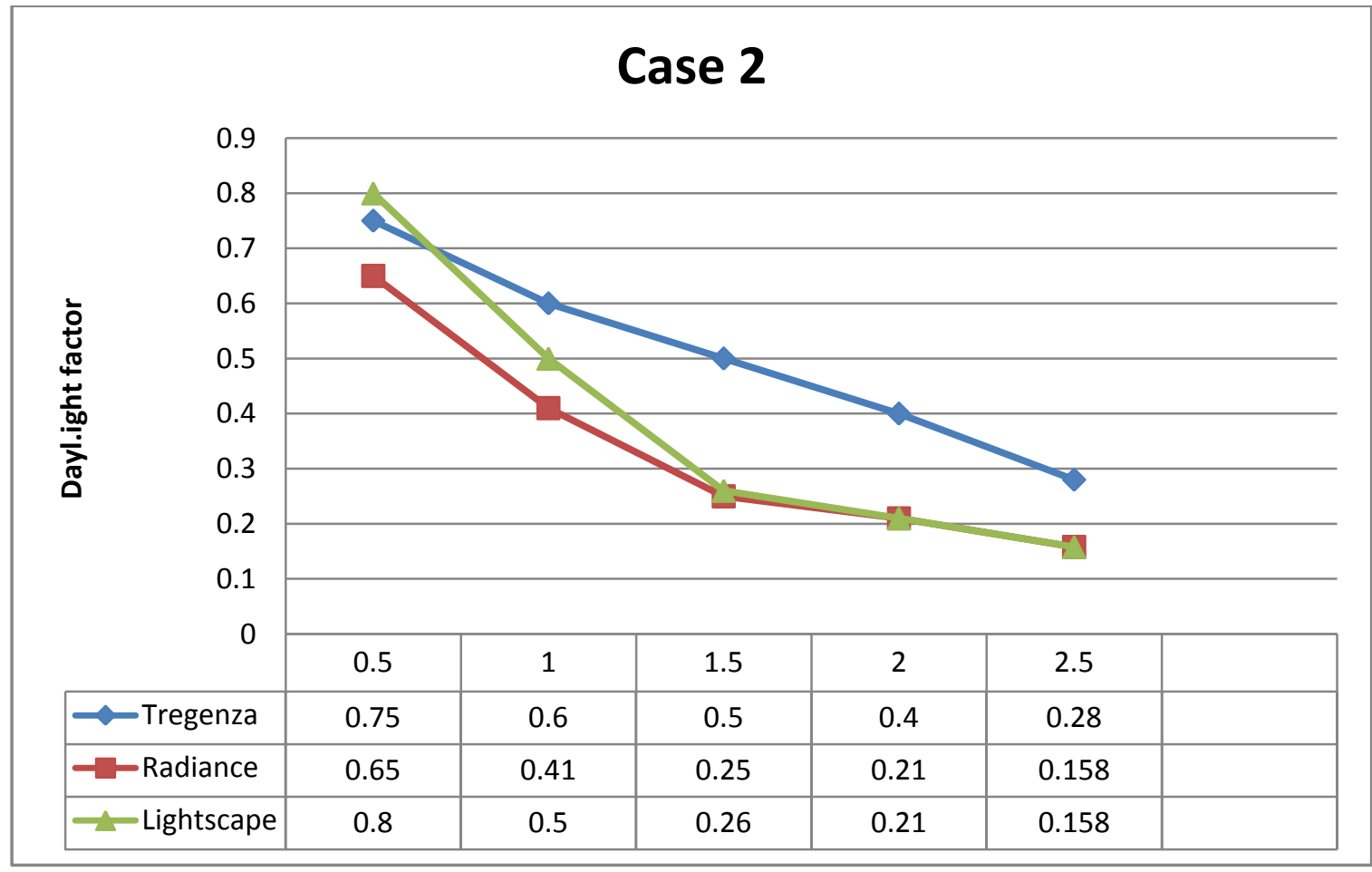

Figure 2: Comparison of horizontal DF for case 2 


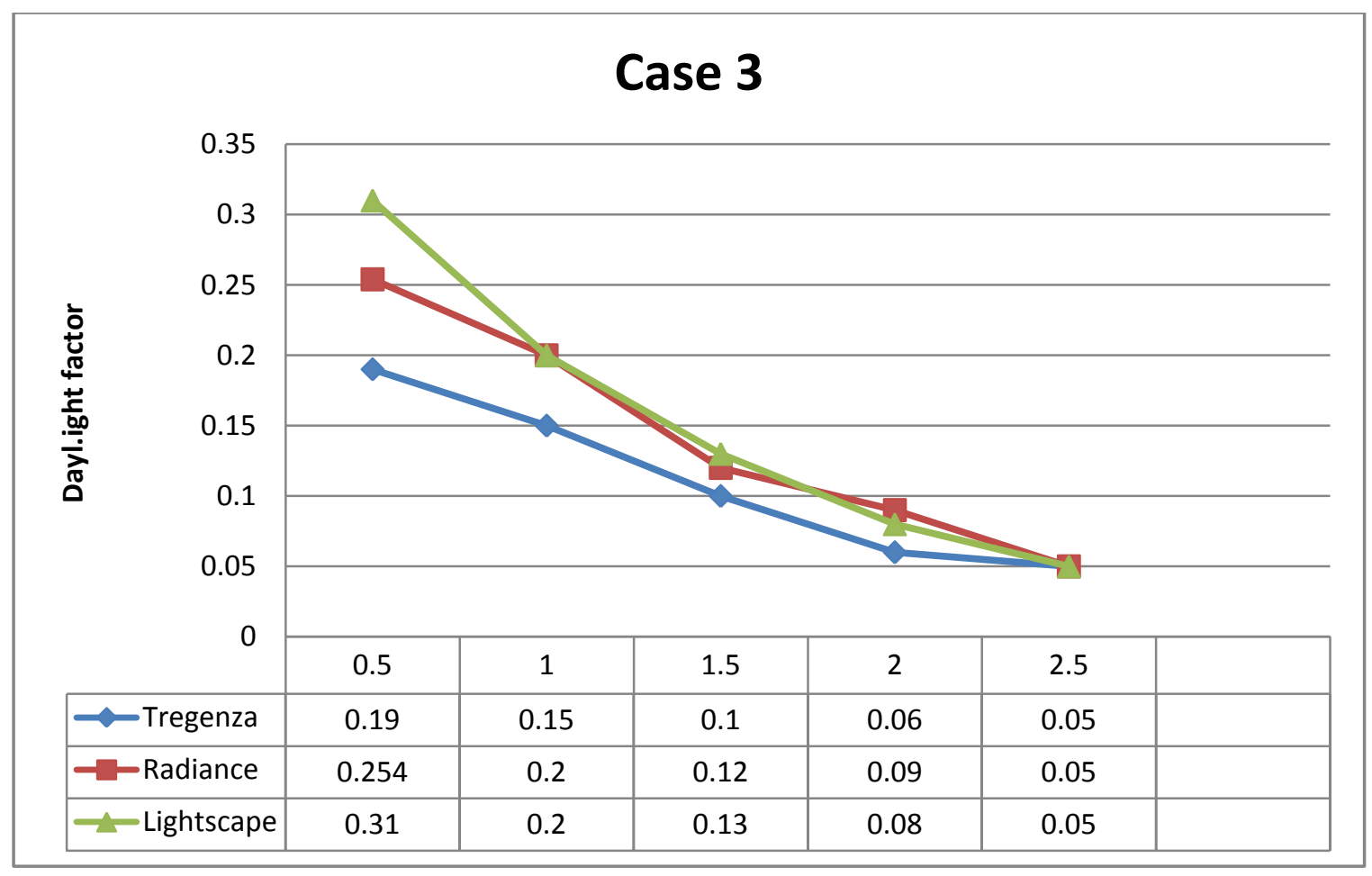

Figure 3: Comparison of Vertica

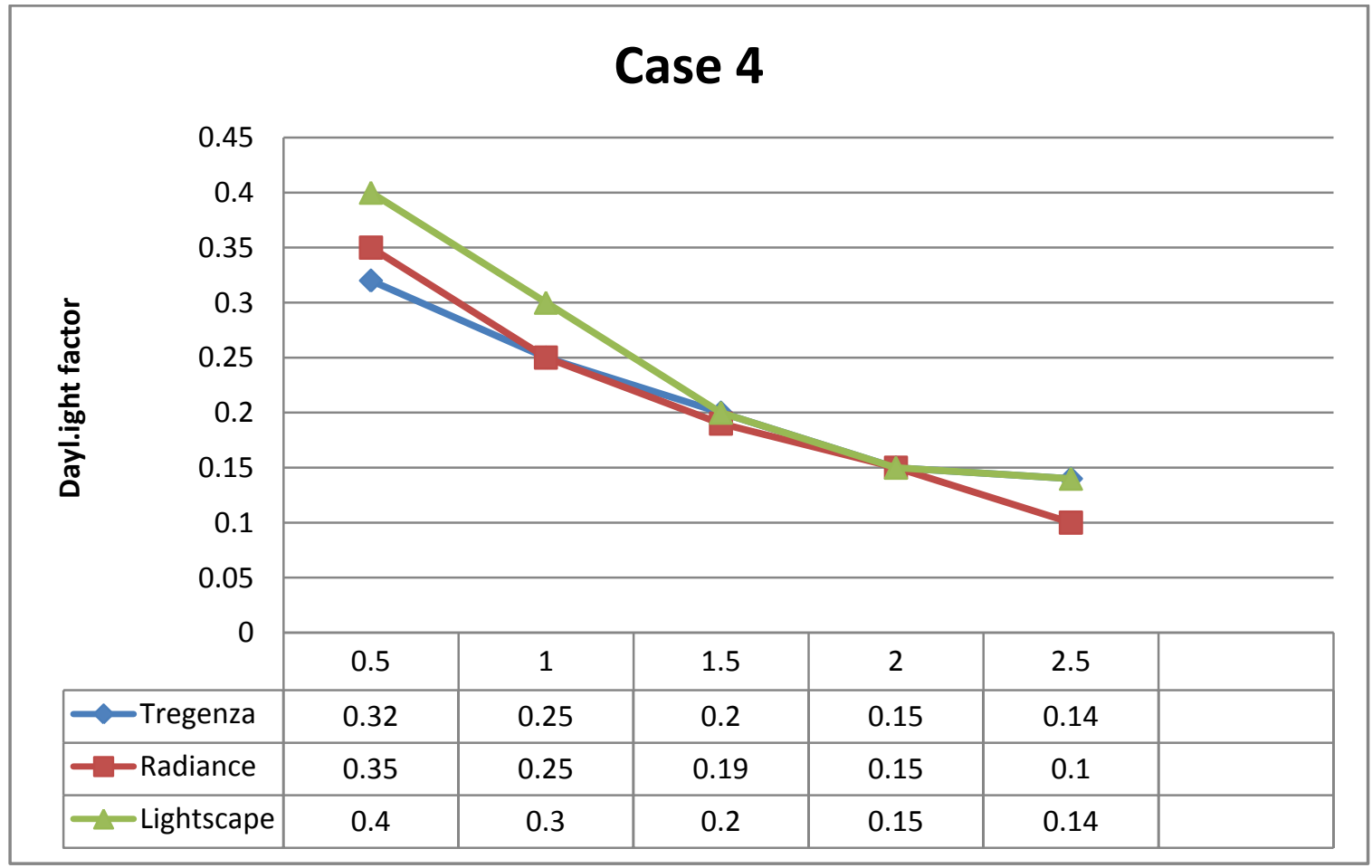

Figure 4: Comparison of Vertical DF for case 2

\section{Following is the analysis of the simulation:-}

1) Trend line indicates an increase in relative differences to reflectance of wall surface. This is as expected owing to deeper reflections put more stress on software

2) Case 1: An overestimation of daylight factors as compared to result from two simulation programme. Relative difference is directly proportional to wellness index.
3) Case 2: Higher the reflectance, greater the difference of radiance. Tregeenza's method showed 30 and Lightscape showed $38 \%$.

4) Case 3 and Case 4 depict the comparison of vertical daylight factors to three methods. Relative difference decrease with wellness index.

5) A more detailed method should be adopted for high 
accuracy results

\section{CONCLUSION}

Ray tracing is an emerging technology and would take time to explore its potential to the fullest but as the technology is gaining momentum; there is a bright future that will be unfolded soon. Cards that will allow global illumination at a lower cost to performance will enhance the effect. New consoles being developed by Microsoft and Sony for implementing real-time ray tracing in video games has a lot of capability but needs improvement over performance. The takeaway is that ray tracing is more HDR than 3D HDR stands for high dynamic range imaging. It is used to reproduce a greater dynamic range of images with different adjustments for brightness and luminosity. Cyberpunk 2077 and nextgeneration consoles are the upcoming milestones to achieve in terms of amount and quality to achieve global illumination. The future of gaming would change the way of how realistic the images, shadow, and lighting would look which would be more visually appealing. Ray tracing will be the most revolutionary technology ever witnessed in the field of animation and graphics.

\section{REFERENCES}

[1] Aubry, M., Maturana, D., Efros, A.A., Russell, B.C., Sivic, J.: Seeing 3D chairs: Exemplar part-based 2D-3D alignment using a large dataset of CAD models. In: CVPR (2014)

[2] Aubry, M., Russell, B.C.: Understanding deep features with computer-generated imagery. In: ICCV

[3] Baker, S., Scharstein, D., Lewis, J.P., Roth, S., Black, M.J., Szeliski, R.: A database and evaluation methodology for optical flow. IJCV 92(1) (2011).

[4] Cordts, M., Omran, M., Ramos, S., Rehfeld, T., Enzweiler, M., Benenson, R., Franke, U., Roth, S., Schiele, B.: The Cityscapes dataset for semantic urban scene understanding. In:CVPR (2016).

[5] Dosovitskiy, A., Fischer, P., Ilg, E., Hausser, P., Hazirbas, C., Golkov, V., vander Smagt, "., Cremers, D., Brox, T.: FlowNet: Learning optical flow with convolutional networks. In: ICCV (2015).

[6] Chen, C., Seff, A., Kornhauser, A.L., Xiao, J.: DeepDriving: Learning affordance for direct perception in autonomous driving. In: ICCV (2015).

[7] Mayer, N., Ilg, E., Hausser, P., Fischer, P., Cremers, D., Dosovitskiy, A., Brox, T.: A large " dataset to train convolutional networks for disparity, optical flow, and scene flow estimation. In: CVPR (2016).

[8] Richter, S.R., Roth, S.: Discriminative shape from shading in uncalibrated illumination. In: CVPR (2015).

[9] Tripathi, S., Belongie, S., Hwang, Y., Nguyen, T.Q.: Semantic video segmentation: Exploring inference efficiency. In: ISOCC (2015).
[10] Xie, J., Kiefel, M., Sun, M.T., Geiger, A.: Semantic instance annotation of street scenes by $3 \mathrm{D}$ to $2 \mathrm{D}$ label transfer. In: CVPR (2016)

[11] Xu, J., Vazquez, D., L ' opez, A.M., Mar' '1n, J., Ponsa, D.: Learning a part-based pedestrian detector in a virtual world. IEEE Transactions on Intelligent Transportation Systems 15(5) (2014)

[12] Yu, F., Koltun, V.: Multi-scale context aggregation by dilated convolutions. In: ICLR (2016)

[13] Lum, E. B., Montrym, J. S., Steiner, W. R., Cobb, J., \& Moreton, H. P. (2018). U.S. Patent No. 9,947,084 Washington, DC: U.S. Patent and Trademark Office.

[14] Wynters, E. (2011). Parallel processing on NVIDIA graphics processing units using CUDA. Journal of Computing Sciences in Colleges, 26(3), 58-66.

[15] Komatitsch, D., Michéa, D., \& Erlebacher, G. (2009). Porting a high-order finite-element earthquake modeling application to NVIDIA graphics cards using CUDA. Journal of Parallel and Distributed Computing, 69(5), 451-460.

[16] Wald, I., Usher, W., Morrical, N., Lediaev, L., \& Pascucci, V. (2019). RTX Beyond Ray Tracing: Exploring the Use of Hardware Ray Tracing Cores for Tet-Mesh Point Location. Proceedings of High Performance Graphics.

[17] Sanzharov, V. V., Gorbonosov, A. I., Frolov, V. A., \& Voloboy, A. G. (2019). Examination of the Nvidia RTX.

[18] Deng Y. et al. Toward real-time ray tracing: A survey on hardware acceleration and microarchitecture tech-niques //ACM Computing Surveys (CSUR). - 2017. - . 50. - №. 4. p. 58.

[19] Gribble C. P., Ramani K. Coherent ray tracing via stream filtering //2008 IEEE Symposium on Interac-tive Ray Tracing. - IEEE, 2008. - p. 59-66.

[20] Hall. D. The AR350: Today's ray trace rendering processor. //Eurographics/SIGGRAPH workshop on Graphics hardware - Hot 3D Session 1, 2001.

[21] Kajiya J. T. The rendering equation //ACM SIG-GRAPH computer graphics. - ACM, 1986. - . 20. -№. 4. - p. 143-150.

[22] Keely S. Reduced precision hardware for ray tracing.//Proc.HPG. - 2014. - p. 29-40.

[23] Kopta D. et al. An energy and bandwidth efficient ray tracing architecture //High-performance Graphics. ACM, 2013. - p. 121-128

[24] Lee W. J. et al. SGRT: A mobile GPU architecture for real-time ray tracing //High-performance graphics conference. - ACM, 2013. - p. 109-119. 